\title{
Negative Capacitance as Performance Booster for Tunnel FETs and MOSFETs: an experimental study
}

\author{
Ali Saeidi, Farzan Jazaeri, Francesco Bellando, Igor Stolichnov, Gia V. Luong, Qing-Tai Zhao, Siegfried Mantl, \\ Christian C. Enz, and Adrian M. Ionescu
}

\begin{abstract}
This letter reports for the first time a full experimental study of performance boosting of Tunnel FETs (TFETs) and MOSFETs by Negative Capacitance (NC) effect. We discuss the importance of capacitance matching between a ferroelectric NC and a device capacitance to achieve hysteretic and non-hysteretic characteristics. PZT ferroelectric capacitors are connected to the gate of three terminals TFETs and MOSFETs and partial or full matching NC conditions for amplification and stability are obtained. First, we demonstrate characteristics of hysteretic and non-hysteretic NC-TFETs. The main performance boosting is obtained for the non-hysteretic NC-TFET, where the on-current is increased by a factor of $500 x$, transconductance is enhanced by three orders of magnitude, and the low slope region is extended. The boosting of performance is moderate in the hysteretic NCTFET. Second, we investigate the impact of the same NC booster on MOSFETs. Subthreshold swing as steep as $4 \mathrm{mV} / \mathrm{dec}$ with a $1.5 \mathrm{~V}$ hysteresis is obtained on a commercial device fabricated in 28nm CMOS technology. Moreover, we demonstrate a nonhysteretic NC-MOSFET with a full matching of capacitances and a reduced subthreshold swing down to $20 \mathrm{mV} / \mathrm{dec}$.
\end{abstract}

Index Terms-negative capacitance, ferroelectric, NC-TFET, NC-FET, hysteretic, non-hysteretic

\section{INTRODUCTION}

$\mathbf{T}$ HE negative capacitance (NC) effect in ferroelectric materials has been proposed to serve as a step-up transformer [1], amplifying the variation of the surface potential $\left(\psi_{s}\right)$ in field effect transistors as a function of the gate voltage $\left(V_{g}\right)$ change. This principle can be used for reducing the supply voltage of electronic switches and for alleviating the increase of the static power consumption in ultra-scaled CMOS devices [2]. The idea is to benefit of NC region of ferroelectric materials [3]. The practical implementation of the $\mathrm{NC}$ involves the series combination of a ferroelectric capacitor, operated in the $\mathrm{NC}$ region [4], with a positive capacitor capable of stabilizing the $\mathrm{NC}$ effect. A negative capacitor in the gate stack can make the total capacitance larger than its classical value, which leads to the decrease of the required $\Delta V_{g}$ to provide the same $\Delta \psi_{s}$. The challenges of ferroelectrics NC integration with conventional transistors are related to achieving simultaneously a matched design of the ferroelectric and in-series stabilizing MOS capacitors [5], [6]. In this context, a significant boosting can occur while the device behavior is still

Ali Saeidi, Francesco Bellando, Igor Stolichnov, and Adrian M. Ionescu are with Laboratory of Micro and Nano-electronic Devices, Ecole Polytechnique FÃl'dÃl'rale de Lausanne, Lausanne, Switzerland.

Farzan Jazaeri and Christian C. Enz are with Laboratory of Integrated Circuits, Ecole Polytechnique FÃl'dÃl'rale de Lausanne, Lausanne, Switzerland.

Gia V. Luong, Qing-Tai Zhao, and Siegfried Mantl are with Peter Grunberg Institut 9 (PGI-9), Forschungszentrum Julich, Julich, Germany.

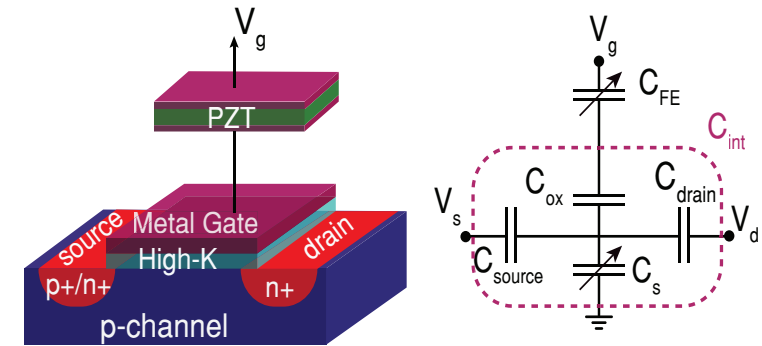

Fig. 1. Experimental configuration of the NC-TFET(p-i-n) /NC-FET(n-i-n) (left) and the capacitance model of the structure (right). $\mathrm{C}_{i n t}$ is the total capacitance of the reference device, including the effect of parasitic capacitors.

non-hysteretic [6]. This paper experimentally investigates the impact of the NC on DC electrical characteristics of Tunnel FETs (TFETs) and MOSFETs. This work demonstrates that the main beneficial effects of the NC on TFETs are the overdrive and transconductance amplification, which addresses exactly the most limiting performances of the current TFETs [2], [7]. Multiple improvements in on-current $\left(\mathrm{I}_{O N}\right)$, transconductance $\left(\mathrm{g}_{m}\right)$, and subthreshold swing (SS), by mean of NC, in a non-hysteretic NC-TFET is achieved. Moreover, a subthermal swing well below $60 \mathrm{mV} / \mathrm{dec}$ is observed in both hysteretic and non-hysteretic NC-FETs.

\section{CAPacitance Matching: Hysteretic AND NON-HysteretiC OPERATION MODES}

A negative capacitance transistor has the unique properties that its gate stack is not passive and includes a mechanism of surface potential amplification [8]. Fig. 1 depicts the equivalent circuit of a MOSFET and of a TFET with a connected NC capacitor $\left(\mathrm{C}_{F E}\right)$ in-series with the gate stack. In both cases, one can define an internal amplification factor,

$$
\beta=\partial V_{i n t} / \partial V_{g}=C_{F E} /\left(C_{F E}+C_{i n t}\right),
$$

where $\mathrm{C}_{F E}$ is the ferroelectric capacitance and $\mathrm{C}_{i n t}$ is the equivalent capacitance of the base transistor from the gate terminal, including all parasitic capacitances. In order to have a sufficient boosting of performance due to the $\mathrm{NC}$ effect, the absolute value of the ferroelectric negative capacitance $\left(\left|C_{F E}\right|\right)$ and the intrinsic gate capacitance $\left(C_{i n t}\right)$ need to be relatively close [5], [9]. In order to achieve a negative capacitance transistor in the non-hysteretic operation mode, the series combination of the ferroelectric negative capacitance and $\mathrm{C}_{\text {int }}$ should remain positive in the whole range of the operation [6]. A negative value of the total capacitance leads 
Hysteretic NC-Tunnel FET

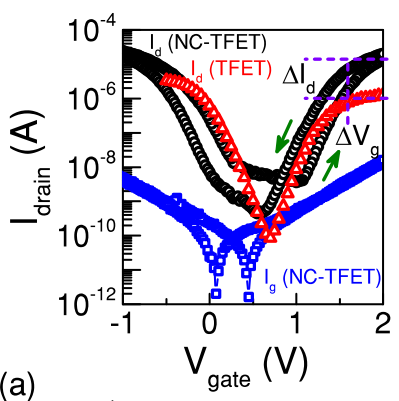

(a)
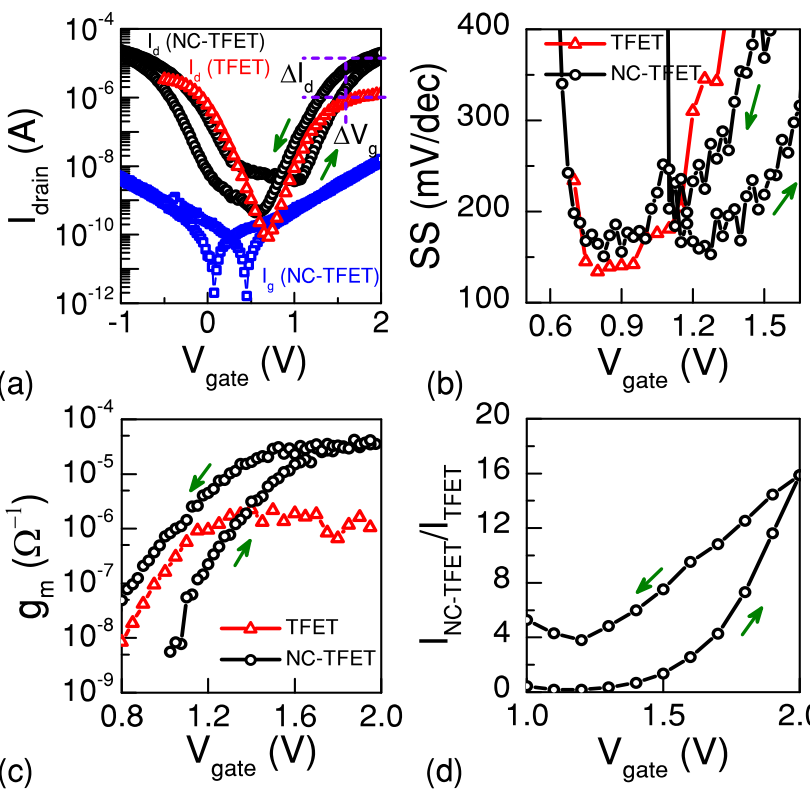

(b)

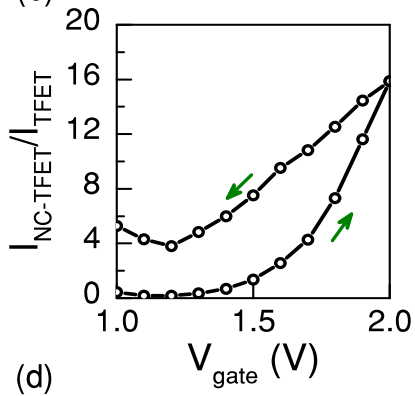

(d)

(c)
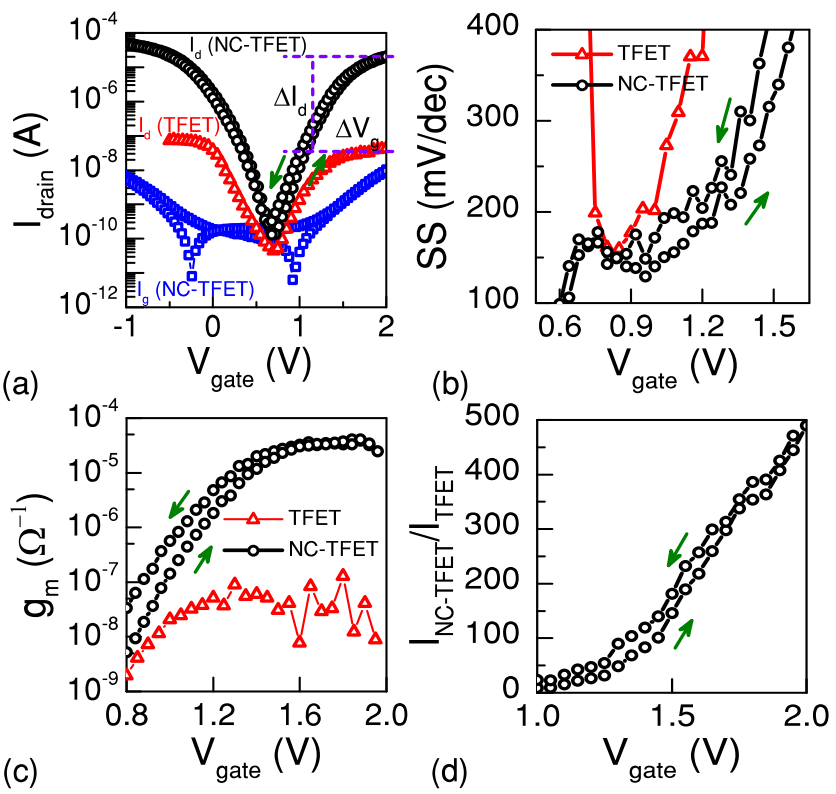

Fig. 2. (a) Transfer characteristic of an $n$-type hysteretic NC-TFET comparing the base device (nanowire array TFETs made of five silicon nanowires with a cross section of $30 \times 5 \mathrm{~nm}^{2}$ and a gate length of $350 \mu \mathrm{m}$ ) where the intrinsic gate capacitance and the $\mathrm{NC}$ are matched only in a limited range of the gate voltage. While NC provides no improvement on the SS of the hysteretic TFET (b), the transconductance (c) and on-current (d) of the device is slightly boosted especially at high $\mathrm{V}_{g}$. The drain voltage is $500 \mathrm{mV}$.

to instability and hysteretic jumps in the polarization that results in a hysteretic transfer characteristic. Accordingly, the matching conditions of a negative capacitance transistor to have a considerable amplification in the non-hysteretic operation of the device can be expressed as

$$
\begin{gathered}
\beta=\partial V_{i n t} / \partial V_{g}=C_{F E} /\left(C_{F E}+C_{i n t}\right) \gg 1, \\
C_{\text {total }}=\left(C_{F E}^{-1}+C_{\text {int }}^{-1}\right)^{-1}>0 .
\end{gathered}
$$

One important consequence of a large internal amplification factor , $\beta$, is the lowering of the body factor, $\mathrm{m}$ :

$$
m=\frac{1}{\beta} \times \frac{\partial V_{i n t}}{\partial \psi_{s}}=\frac{1}{\beta} \times\left(1+\frac{C_{S}}{C_{O X}}\right) \ll 1 .
$$

where $\mathrm{C}_{S}$ and $\mathrm{C}_{O X}$ are the semiconductor and gate oxide capacitances respectively.

\section{Negative Capacitance Tunnel FET}

In this section, we report experimental results obtained by connecting an external PZT capacitor to the gate of an $n$ type strained Silicon-NanoWire (Si-NW) array TFET. The device consists of five common gate nanowires with a cross section of $30 \times 5 \mathrm{~nm}^{2}$ and a gate length of $350 \mu \mathrm{m}$. This external connection offers the advantage of testing many PZT capacitors and transistors until the best matching is obtained. For this experiment, $50 \mathrm{~nm}$ of $\mathrm{Pb}\left(Z r_{43}, \mathrm{Ti}_{57}\right) \mathrm{O}_{3}$ (PZT) ferroelectric film has been grown via the chemical solution deposition root on a Pt-coated silicon wafer. The

Fig. 3. (a) An outstanding performance boosting is simultaneously achieved together with an improvement of the swing, where the ferroelectric NC and the gate capacitance of the transistor (nanowire array TFETs made of five silicon nanowires with a cross section of $30 \times 5 \mathrm{~nm}^{2}$ and a gate length of $350 \mu \mathrm{m}$ ) are matched in the whole range of the gate voltage. An extended low slope region (b), transconductance boosting up to 3 orders of magnitude (c), and up to 500x current improvement are obtained $\left(\mathrm{V}_{\text {drain }}=500 \mathrm{mV}\right)$.

stack of $\mathrm{Pt}(100 \mathrm{~nm}) / \mathrm{TiO}_{2}(30 \mathrm{~nm})$ has been sputtered on a $\mathrm{SiO}_{2}(500 \mathrm{~nm}) / \mathrm{Si}$ wafer at $300^{\circ} \mathrm{C}$ [10]. The relative permittivity, coercive field, and remanent polarization of $220-240$, $260 \mathrm{kV} / \mathrm{cm}$, and $32 \mu \mathrm{C} / \mathrm{cm}^{2}$ have been measured through the $P t$ top electrode. Generally, high-quality epitaxial layers are employed for negative capacitance devices. The demonstration of NC effect using a polycrystalline ferroelectric layer constitutes a significant step towards the integration of $\mathrm{NC}$ gates in CMOS technology. In fact, fabrication of epitaxial perovskite layers on silicon is an extremely challenging task, whereas polycrystalline ferroelectrics (like PZT) can be integrated.

Fig. 2 and Fig. 3 depict experimental performance boosting, reached on relatively fair homojunction TFETs with an oncurrent of $0.1 \mu \mathrm{A}\left(V_{g}=2 \mathrm{~V}\right.$ and $\left.V_{d}=0.5 \mathrm{~V}\right)$ and a swing is in the order of $100-150 \mathrm{mV} / \mathrm{dec}$, in hysteretic and non-hysteretic modes of operation. Fig. 2-a reports a hysteretic characteristic where the values of $\mathrm{C}_{P Z T}$ and $\mathrm{C}_{i n t}$ are verifying conditions (2) and (3) only in a limited range of operation (for gate voltages larger than the threshold voltage). In this case, the performance boosting is narrowed down to a reduced region. Figs. 2-b, 2-c, and 2-d summarize the effects of the PZT negative capacitance on the SS (no improvement), $\mathrm{g}_{m}$ (1 to 2 orders of magnitude), and $\mathrm{I}_{O N}$ (a factor of up to 16x). Such a hysteretic NC Tunnel FET can still be considered for low power hysteretic logic applications or as a 1 TFET ferroelectric memory cell with improved on-current performance [11].

In the other case, we exploit another NC-TFET in the same configuration with a different TFET and PZT capacitor, matching in the entire range of the gate voltage (Fig. 3). The area of 
the utilized PZT capacitor is half of the previous experiment that is providing lower negative capacitance. Therefore, the total capacitance of the structure remains positive and the non-hysteretic operation of the device can be achieved. In this late case, a significant double improvement in the SS and overdrive is observed in a hysteresis-free NC-TFET. The NC effect appears to reduce only moderately the subthreshold swing and extends the region of low slope values (Fig. 3b); this is probably due to the low $\beta$ value in the matching equation (1). Moreover, due to the poor swing $(150 \mathrm{mV} / \mathrm{dec})$ of the initial TFET, the represented NC-TFET does not reach sub-thermionic slope values. Other limiting factors of TFETs are their poor transconductance and on-current. Fig. 3-c shows the obtained boosting of $\mathrm{g}_{m}$, ranging from 10 to $5 \times 10^{3}$ times of its original value, due to the differential amplification of the surface potential by NC effect. The on-current is boosted over the whole operation range, reaching a factor of about 500x at the maximum gate voltage (Fig. 3-d). This is reflected by a body factor reduction below 1, acting as a performance booster in the region of low on-current [12]. In equation (2), at higher $\mathrm{V}_{g}$, the $\mathrm{C}_{i n t}$ is close to $\mathrm{C}_{F E}$ and $\beta \gg 1$. Consequently, the body factor, $\mathrm{m}$, becomes significantly smaller than 1 (see equation 4). It follows that the gate voltage can be reduced by $65 \%$, maintaining the same level of the output current [13]. The non-hysteretic NC-TFET could be a potential candidate for low-power steep slope switches for both analog and digital applications. It should be remarked that the leakage and charge trapping mechanisms are neglected due to the fact that the gate leakage in both hysteretic and non-hysteretic devices is systematically lower than the on-current.

\section{Negative CAPacitance MOSFET}

The same negative capacitance booster is externally connected to the gate of various $n$-MOSFETs that are manufactured in 28nm CMOS technology and also larger devices which are fabricated on an SOI substrate. Different combinations of PZT capacitors and MOSFETs have been examined to obtain NC-FETs in hysteretic and non-hysteretic operation modes. The experiments are conducted with the same PZT capacitors reported in the previous section. All measurements have been carried out at low drain voltages due to the fact that a high drain voltage provides a non-uniform potential profile along the channel and the NC effect cannot be observed [14].

Fig. 4-a shows the hysteretic behavior of an NC-FET with a $28 \mathrm{~nm}$ CMOS process MOSFET ( $W=333 \mu \mathrm{m}, L=30 \mathrm{~nm}$ ) and a PZT capacitor with an area of $30 \mu m \times 30 \mu m$. In this late case, a swing of $4 \mathrm{mV} / \mathrm{dec}$ is achieved over six decades of current in the linear operation mode $\left(V_{d}=100 \mathrm{mV}\right)$. It should be remarked that due to the small intrinsic gate capacitance of $28 \mathrm{~nm}$ commercial MOSFETs, the total capacitance of the NC-FET cannot be positive in the whole range of experiment $\left(C_{\text {total }}>0\right.$ condition is not fulfilled).Therefore, the NC-FET is not stable and provide hysteresis [6].

MOSFETs on an SOI silicon wafer with relatively large dimensions are fabricated to fulfill the condition for nonhysteretic operation of a non-hysteretic NC-FET. The devices are built on a $p$-type SOI substrate with $88 \mathrm{~nm}$ of epitaxial

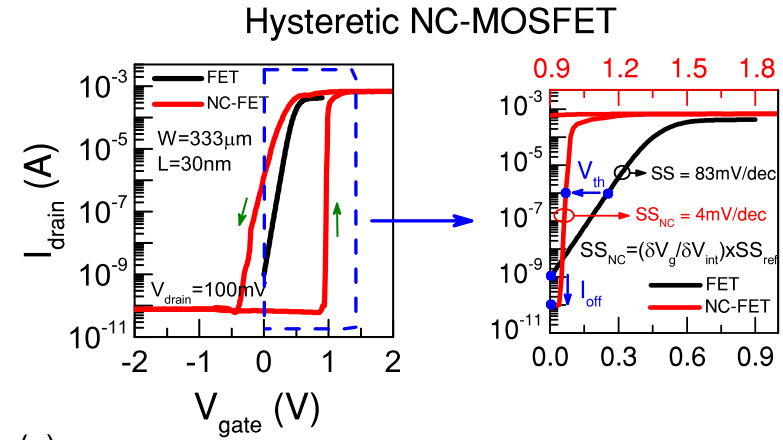

(a)

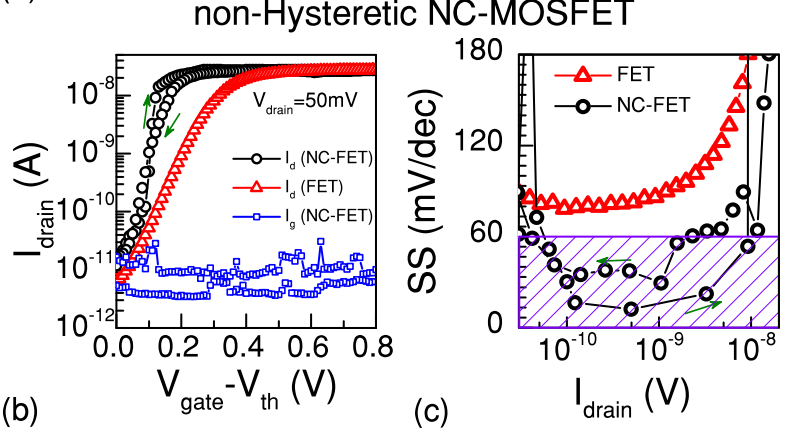

Fig. 4. (a) Effect of the NC on DC characteristics of an $n$-type commercial MOSFET fabricated in 28nm CMOS technology (W=333 $\mu \mathrm{m}, \mathrm{L}=30 \mathrm{~nm})$. Subthreshold swing of $4 \mathrm{mV} / \mathrm{dec}$ over six decades of current is achieved together with a $1.5 \mathrm{~V}$ hysteresis. A MOSFET with approximately 4x larger gate area/capacitance $(\mathrm{W}=19 \mu \mathrm{m}, \mathrm{L}=2 \mu \mathrm{m})$ fabricated on an SOI substrate with a full matching of the MOS and ferroelectric NC is demonstrated in (b) where a sub-thermal swing is achieved in a hysteresis-free NC-FET.

silicon (thinned down to $30 \mathrm{~nm}$ ) and $145 \mathrm{~nm}$ of BOX. A $3 \mathrm{~nm}$ layer of $\mathrm{HfO}_{2}$ has been deposited by ALD on an ultra thin layer of $\mathrm{SiO}_{2}$ as the gate dielectric. Fig. 4-b demonstrates the transfer characteristic of a hysteresis-free NC-FET with a drain voltage of $50 \mathrm{mV}$. The gate width and gate length of the reference MOSFET are respectively $19 \mu \mathrm{m}$ and $2 \mu \mathrm{m}$ while the PZT capacitor has an area of $20 \mu \mathrm{m} \times 20 \mu \mathrm{m}$. A significant enhancement in the SS of the device is obtained when the ferroelectric and gate capacitances are fully matched so that a non-hysteretic NC operation can be achieved. The ferroelectric provides an effective $\mathrm{NC}$ in the subthreshold region of MOSFETs $(\beta<1)$ which results in a sub-thermal swing $(20 \mathrm{mV} / \mathrm{dec})$ [15].

\section{CONClusion}

The hysteretic and non-hysteretic behavior of negative capacitance tunnel FETs and MOSFETs are experimentally investigated in this letter. It has been evidenced that the negative capacitance can be efficiently utilized to significantly enhance the on-current and transconductance of TFETs in the overdrive region while it improves the subthreshold swing of MOSFETs. A matching condition is proposed and satisfied between the ferroelectric and MOS capacitors for the nonhysteretic operation of negative capacitance transistors.

\section{ACKNOWLEDGMENT}

The authors would like to thank the Swiss National Science Foundation (grant number 149495) and, in part, the ERC Advanced Grant Milli-Tech for providing the financial support. 


\section{REFERENCES}

[1] S. Salahuddin and S. Datta, "Use of negative capacitance to provide voltage amplification for low power nanoscale devices," Nano letters, vol. 8, no. 2, pp. 405-410, 2008, DOI: 10.1021/n1071804g.

[2] A. M. Ionescu, L. De Michielis, N. Dagtekin, G. Salvatore, J. Cao, A. Rusu, and S. Bartsch, "Ultra low power: Emerging devices and their benefits for integrated circuits," in Electron Devices Meeting (IEDM), 2011 IEEE International. IEEE, 2011, DOI: 10.1109/IEDM.2011.6131563, pp. 16-1.

[3] A. I. Khan et al., "Negative capacitance in a ferroelectric capacitor," Nature materials, vol. 14, no. 2, pp. 182-186, 2015, DOI: $10.1038 / \mathrm{nmat} 4148$.

[4] D. J. Appleby, N. K. Ponon, K. S. Kwa, B. Zou, P. K. Petrov, T. Wang, N. M. Alford, and A. O Neill, "Experimental observation of negative capacitance in ferroelectrics at room temperature," Nano letters, vol. 14, no. 7, pp. 3864-3868, 2014, DOI: 10.1021/n15017255.

[5] A. Rusu, A. Saeidi, and A. M. Ionescu, "Condition for the negative capacitance effect in metal-ferroelectric-insulator-semiconductor devices," Nanotechnology, vol. 27, no. 11, p. 115201, 2016, DOI: 10.1088/0957-4484/27/11/115201.

[6] A. Saeidi, F. Jazaeri, I. Stolichnov, and A. M. Ionescu, "Double-Gate Negative-Capacitance MOSFET With PZT Gate-Stack on Ultra Thin Body SOI: An Experimentally Calibrated Simulation Study of Device Performance," IEEE Transactions on Electron Devices, vol. 63, no. 12, pp. 4678-4684, 2016, DOI: 10.1109/TED.2016.2616035.

[7] Q. Zhang, W. Zhao, and A. Seabaugh, "Low-subthreshold-swing tunnel transistors," IEEE Electron Device Letters, vol. 27, no. 4, pp. 297-300, 2006, DOI: 10.1109/LED.2006.871855.

[8] S. Salahuddin and S. Datta, "Can the subthreshold swing in a classical fet be lowered below $60 \mathrm{mv} /$ decade?" in Electron Devices Meeting, 2008. IEDM 2008. IEEE International. IEEE, 2008, DOI: 10.1109/IEDM.2008.4796789, pp. 1-4.

[9] A. I. Khan, C. W. Yeung, C. Hu, and S. Salahuddin, "Ferroelectric negative capacitance MOSFET: Capacitance tuning \& antiferroelectric operation," in Electron Devices Meeting (IEDM), 2011 IEEE International. IEEE, 2011, DOI: 10.1109/IEDM.2011.6131532, pp. 11-3.

[10] J. Lee, C. Choi, B. Park, T. Noh, and J. Lee, "Built-in voltages and asymmetric polarization switching in $\mathrm{Pb}(\mathrm{Zr}, \mathrm{Ti}) \mathrm{O}_{3}$ thin film capacitors," Applied physics letters, vol. 72, no. 25, pp. 3380-3382, 1998, DOI: http://dx.doi.org/10.1063/1.121610.

[11] A. Saeidi, A. Biswas, and A. M. Ionescu, "Modeling and simulation of low power ferroelectric non-volatile memory tunnel field effect transistors using silicon-doped hafnium oxide as gate dielectric," Solid-State Electronics, vol. 124, pp. 16-23, 2016, DOI: https://doi.org/10.1016/j.sse.2016.07.025.

[12] A. M. Ionescu and H. Riel, "Tunnel field-effect transistors as energyefficient electronic switches," Nature, vol. 479, no. 7373, pp. 329-337, 2011, DOI: 10.1038/nature10679.

[13] C. Wu, R. Huang, Q. Huang, C. Wang, J. Wang, and Y. Wang, "An analytical surface potential model accounting for the dual-modulation effects in tunnel FETs," IEEE Transactions on Electron Devices, vol. 61, no. 8, pp. 2690-2696, 2014, DOI: 10.1109/TED.2014.2329372.

[14] A. Rusu, G. A. Salvatore, D. Jimenez, and A. M. Ionescu, "Metalferroelectric-metal-oxide-semiconductor field effect transistor with sub$60 \mathrm{mv} /$ decade subthreshold swing and internal voltage amplification," in Electron Devices Meeting (IEDM), 2010 IEEE International. IEEE, 2010, DOI: 10.1109/IEDM.2010.5703374, pp. 16-3.

[15] J. Jo, W. Y. Choi, J.-D. Park, J. W. Shim, H.-Y. Yu, and C. Shin, "Negative capacitance in organic/ferroelectric capacitor to implement steep switching MOS devices," Nano letters, vol. 15, no. 7, pp. 45534556, 2015, DOI: 10.1021/acs.nanolett.5b01130. 